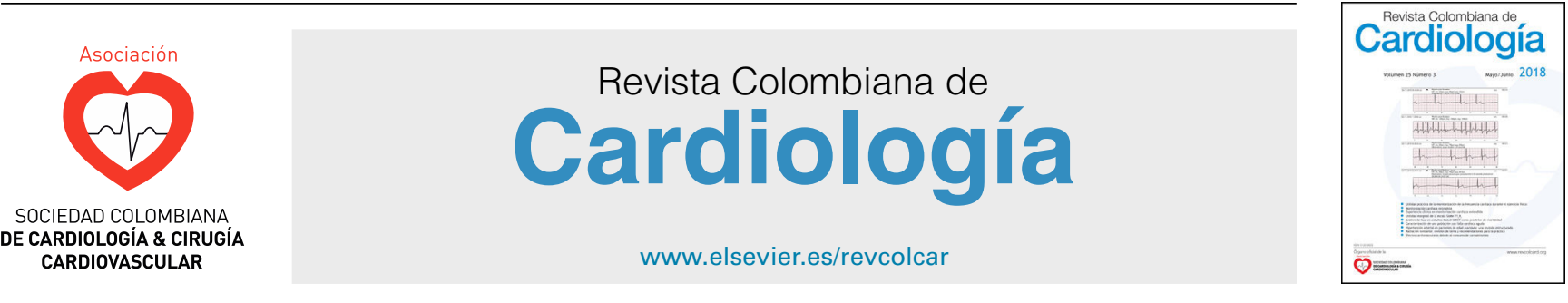

ADULT CARDIOLOGY - ORIGINAL ARTICLE

\title{
Quality of life assessment after successful percutaneous transmitral commissurotomy and ascertain its relationship with gender
}

\author{
Hammad Shah $^{\mathrm{a}, *}$, Momin Salahuddin $^{\mathrm{a}}$, Muneeb Ullah Jan ${ }^{\mathrm{b}}$, Afrasyab Altaf $^{\mathrm{a}}$ \\ a Cardiology Department, Rehman Medical Institute, Peshawar, Pakistan \\ ${ }^{\mathrm{b}}$ Department of Medicine, Khyber Teaching Hospital, Peshawar, Pakistan
}

Received 29 September 2018; accepted 17 January 2019

Available online 12 July 2019

\section{KEYWORDS \\ Quality of life; \\ WHOQol-100; \\ Percutaneous \\ transmitral \\ commissurotomy; \\ Gender; \\ Mitral stenosis; \\ Mitral valve area}

\begin{abstract}
Background: percutaneous transmitral commissurotomy has become an ideal treatment option for mitral stenosis due to its less adverse events and more favorable outcomes. Patients improve symptomatically after percutaneous transmitral commissurotomy but we have minimal available data about the quality of life after percutaneous transmitral commissurotomy.

Objective: to assess the quality of life after percutaneous transmitral commissurotomy through WHOQol scoring covering different aspects of life, to determine its correlation with a net gain in mitral valve area (MVA) and to ascertain its association with gender.

Methods: it was a prospective cohort study carried out for a period of 15 months. A total of 100 patients with mitral stenosis who had successful percutaneous transmitral commissurotomy done were enrolled in the study. Among the total included, 4 patients were lost to follow up and data were collected from 96 patients. Quality of life was assessed before the procedure, at 1 month and 3 months follow up after percutaneous transmitral commissurotomy using WHOQol scoring questionnaire.

Results: among 96 patients, 64 (67\%) were females and 32 (33\%) were males. WHOQol scoring improved significantly after percutaneous transmitral commissurotomy from $32.8 \pm 8.9$ to $54.6 \pm 11.2$ and $62.8 \pm 9.7$, after one month and 3 months respectively. There was a significant association between net gain of MVA and WHOQol scoring with an R value of 0.46 and $p$ value of 0.03 . There was no difference in group comparison of all the six domains between male and female patients.
\end{abstract}

\footnotetext{
* Corresponding author.

E-mail address: drhammadshah@gmail.com (H. Shah).
} 
Conclusion: successful percutaneous transmitral commissurotomy improves the quality of life in mitral stenosis patients regardless of their gender and has a positive correlation with a net gain in mitral valve area.

(c) 2019 Sociedad Colombiana de Cardiología y Cirugía Cardiovascular. Published by Elsevier España, S.L.U. This is an open access article under the CC BY-NC-ND license (http:// creativecommons.org/licenses/by-nc-nd/4.0/).

\section{PALABRAS CLAVE}

Calidad de vida; WHOQol-100; Comisurotomía mitral percutánea; Sexo; Estenosis mitral; Área valvular mitral

\section{Introduction}

There are multiple causes of mitral valve stenosis which include rheumatic heart disease, congenital heart disease, infective endocarditis, calcification, rare causes like SLE, carcinoid syndrome, Whipple disease, Fabry disease and rheumatoid arthritis. Among all, rheumatic heart disease (RHD) is the most abundant and leading cause of mitral valve scarring. ${ }^{1}$ The prevalence of RHD has declined globally but in underdeveloped countries, it is still highly endemic and most common cause of cardiovascular morbidity. ${ }^{2}$ The mitral valve is the most commonly involved valve in RHD. ${ }^{3,4}$ has a valve area of $4-6 \mathrm{~cm}^{2}$ and causes symptoms when valve area becomes less than $2 \mathrm{~cm}^{2}$. It is the most common valvular lesion of pregnancy and is poorly tolerated. ${ }^{5}$ Transthoracic echocardiography is the investigation of choice to diagnose mitral valve stenosis and to evaluate its area. ${ }^{6}$ Symptomatic mitral stenosis has different treatment options but percutaneous transmitral commissurotomy is the accepted treatment modality of choice in rheumatic mitral stenosis with favorable valve morphology ${ }^{7}$. The treatment of percutaneous transmitral commissurotomy started in 1980 and has evolved from double balloon to single Inoue balloon ${ }^{8}$. The efficacy results of the single balloon and double balloon are similar, but the single balloon has an added advantage of being more simple and having a more favorable safety profile. ${ }^{9}$ The percutaneous transmitral commissurotomy can provide symptomatic relief to patients of mitral stenosis who are planning to become pregnant and can be performed safely in the second trimester of pregnancy ${ }^{10}$. It can also treat mitral stenosis due to RHD, calcification or any other etiology effectively ${ }^{11}$.

The World health organization (WHO) defines health as not merely the absence of disease or infirmity but a state of mental, physical and social well-being ${ }^{12}$. The WHOQol 100 questionnaire was designed to assess the quality of life in six different domains ${ }^{13}$. This questionnaire was used to assess the quality of life in patients with chronic diseases 
in China ${ }^{14}$, but there is limited available data to evaluate the quality of life after percutaneous transmitral commissurotomy.

This study is therefore aimed to assess the quality of life after percutaneous transmitral commissurotomy using multimodal domains of WHOQol 100 questionnaire, determine its correlation with a net gain in mitral valve area and ascertain its association with gender.

\section{Methods and materials}

It was a prospective cohort study carried out at the cardiology department of Rehman Medical Institute. Using a universal sampling technique, a total of 100 patients were enrolled over a period of 15 months. It included all those patients who had successful percutaneous transmitral commissurotomy done for mitral stenosis and had post procedure mitral valve area of more than $1.5 \mathrm{~cm} 2$ on transthoracic echo. Patients with age, more than 20 years and less than 70 years, from both genders, multiple geographic locations, different socioeconomic status and diverse ethnic backgrounds were part of the study population.

Patient with aortic stenosis, hypertrophied obstructive cardiomyopathy (HOCM), severe mitral regurge $2+$, severe aortic regurge $2+$, new-onset atrial fibrillation, heart failure with NYHA class III/IV, myocardial infarction with regional wall motion abnormalities on echo, ejection fraction less than $40 \%$, chronic renal disease with ultrasonographic evidence of renal parenchymal disease, chronic liver disease with ultrasonographic evidence of liver fibrosis, pericarditis and chronic obstructive pulmonary disease (COPD) with restrictive lung disease on spirometry were excluded from the study population because all of these conditions can invariably affect the quality of life. Informed written consent was obtained and confidentiality of the patients was maintained. Data was collected using WHOQoL-100 questionnaire before the procedure, after 01 months and 03 months of follow up. Ethical approval was obtained from the research and ethical unit (REU) of Rehman Medical Institute (RMI). Our study abided by the declaration of Helsinki.

The percutaneous transmitral commissurotomy procedure was done using femoral transvenous antegrade approach and mitral valve area was calculated noninvasively by transthoracic echo. Single Inoue balloon was used and balloon size was determined by height in the $\mathrm{cm} / 10+10$ formula. WHOQOL-100 questionnaire was completed, by asking questions using 24 facets in 6 domains along with 4 general facets and marking it from 0-5 with 0 being the worst and 5 being the best to assess the quality of life. A transformed total score was calculated at the end of the questionnaire to which each facet contributed equally and had a range from 0-100. High score denoted a better quality of life. Mitral valve area was measured echocardiographically by doing planimetry in parasternal-short axis view, from tips of mitral leaflets. Right ventricular systolic pressure was calculated by determining the pressure gradient between the right atrium and right ventricle from Doppler tricuspid jet in apical four chamber view using Bernoulli law $\mathrm{P}=4 \mathrm{~V}^{2}$ and adding right atrial pressure determined from inferior vena cava collapsibility to it.
Table 1 Baseline characteristics of study population.

\begin{tabular}{ll}
\hline Category & Frequency \\
\hline Gender & \\
$\quad$ Male & $32(33 \%)$ \\
Female & $64(67 \%)$ \\
Smokers & $37(39 \%)$ \\
Hypertension & $32(33 \%)$ \\
Diabetes mellitus & $39(41 \%)$ \\
Atrial fibrillation & $51(53 \%)$ \\
Obese & $2(2.2 \%)$ \\
Overweight & $3(3.1 \%)$ \\
Medications & \\
Aspirin & \\
Diuretics & $90(93.8 \%)$ \\
Betablockers & $82(85.4 \%)$ \\
CCB & $53(55.2 \%)$ \\
Digoxin & $27(28.1 \%)$ \\
Amiodarone & $16(16.7 \%)$ \\
Nitrates & $12(12.5 \%)$ \\
Anti-coagulants & $16(16.7 \%)$ \\
ACEl/ARBs & $23(24 \%)$ \\
\hline
\end{tabular}

\section{Statistical analysis}

The data were analyzed using SPSS 20. Shapiro-Wilk test was used to check the distribution of data. Continuous variables were determined as mean \pm standard deviation while categorical variables were determined as percentages and frequencies. T Test and ANOVA were used to compare the means. Pearson correlation coefficients were used to assess the correlation and relationship strength between continuous variables. A p value of less than 0.05 was considered significant.

\section{Results}

A total of 100 patients were enrolled in the study population who underwent percutaneous transmitral commissurotomy for mitral stenosis and had a valve area of more than $1.5 \mathrm{~cm}^{2}$ on transthoracic echo, done after the procedure. Among the total, 4 patients were lost to follow up. Data were collected from 96 patients before the procedure and after the procedure over one month and 3 months follow up period which showed a mean age of $30.2 \pm 3.8$ years. Baseline characteristics are shown in Table 1.

The mean value of four main variables was compared before the procedure after the procedure at one month and three months follow up period. There was a significant improvement in all the main variables, when measured before the procedure, at one month and three months follow up period on paired t-test with $\mathrm{p}<0.001$ as shown in the Table 2 below.

Chi square test was used to ascertain correlation between the WHOQol-100 scoring and increase in mitral valve area which showed statistical significance with $p$ value of 0.03 . Pearson correlation although positive, had a moderately 
Table 2 Comparison of mean before and after percutaneous transmitral commissurotomy at 1 and 3 month follow up.

\begin{tabular}{llll}
\hline & $\begin{array}{l}\text { Before percutaneous } \\
\text { transmitral commissurotomy } \\
\text { mean } \pm \text { SD }\end{array}$ & $\begin{array}{l}\text { After 01 month } \\
\text { mean } \pm \text { SD }\end{array}$ & $\begin{array}{l}\text { After 03 months } \\
\text { mean } \pm \text { SD }\end{array}$ \\
\hline WHOQoL 100 score & $32.8 \pm 8.9$ & $54.6 \pm 11.2$ & $62.8 \pm 9.7$ \\
Mitral valve area cm & $0.8 \pm 0.67$ & $1.73 \pm 0.89$ & $1.91 \pm 1.28$ \\
RVSP mm Hg & $62.24 \pm 14.19$ & $33 \pm 8.12$ & $32 \pm 7.39$ \\
NYHA class & $2.9 \pm 0.38$ & $2.1 \pm 0.42$ & $1.1 \pm 0.35<0.001$ \\
\hline
\end{tabular}

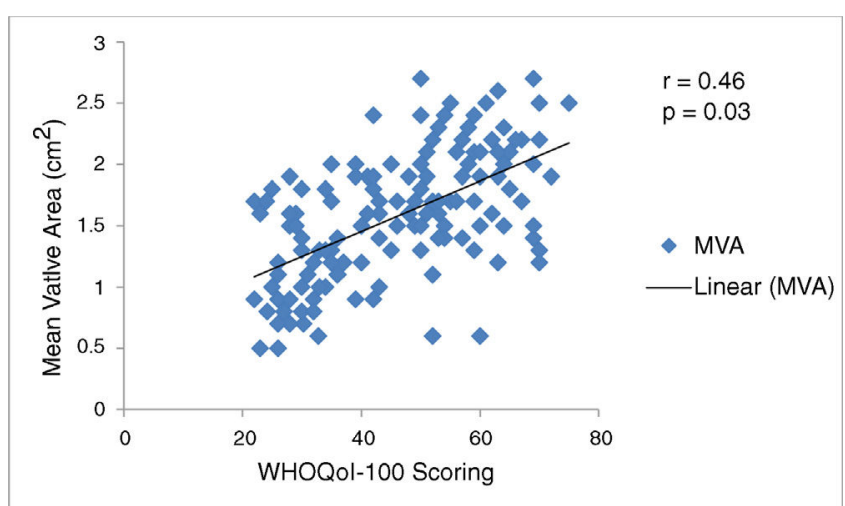

Figure 1 Scatterplot graph showing correlation between MVA and WHOQoL-100 scoring.

positive coefficient( $r$ ) value of 0.46 as shown in the scatterplot graph in Figure 1.

It was compared the group mean of six different domains of WHOQol-100 questionnaire with gender difference before percutaneous transmitral commissurotomy and after three months of the procedure. There was no significant group difference between male and female patients quality of life in all the six domains on ANOVA analysis as shown in Table 3.

\section{Discussion}

Health is not only an absence of disease or disability but is defined as a condition of mental, physical and social wellbeing by WHO. ${ }^{12}$ Quality of life is assessed by different tools but WHOQol-100 is validated as authentic one to assess the quality of life by $\mathrm{WHO}^{13}$ and is tested in patients with longstanding chronic diseases. ${ }^{14}$ Mitral stenosis is a chronic ailment which badly affects the quality of life. ${ }^{3,4}$ Percutaneous transmitral commissurotomy has evolved as an ideal treatment of choice not only in routine patients but can be carried out safely in pregnant patients as well. ${ }^{5}$ We are lacking data about its correlation with quality of life. This study was therefore aimed to assess the physical, social and mental wellbeing of patients after percutaneous transmitral commissurotomy using multimodal domains of WHOQol 100 questionnaire, to determine its correlation with a net gain in mitral valve area and ascertain its association with gender.

In this study, about $67 \%$ of the patients were females as compared to $33 \%$ males which are comparable with the results of Movahed et al. ${ }^{15}$ study in which mitral stenosis was more common in females, however, the cause of association was unknown. Khan et al. ${ }^{16}$ found, that symptomatic improvement occurs after percutaneous transmitral commissurotomy by improvement in NYHA score. In our study, the NYHA score improved from $2.9 \pm 0.38$ to $2.1 \pm 0.42$

Table 3 Group correlation between gender with WHOQol-100 domains before percutaneous transmitral commissurotomy and after 3 months.

\begin{tabular}{lllll}
\hline WHOQol-100 Domain & Status & Male & Female & P value \\
\hline Physical health & Before percutaneous transmitral commissurotomy & $24.7 \pm 6.1$ & $25.5 \pm 7.2$ & 0.85 \\
& After 3 Months & $57.9 \pm 10.9$ & $59.1 \pm 10.3$ & 0.93 \\
Psychological & Before percutaneous transmitral commissurotomy & $32.1 \pm 8.5$ & $31.2 \pm 9.2$ & 0.89 \\
& After 3 Months & $60.2 \pm 11.2$ & $58.9 \pm 10.9$ & 0.96 \\
Level of & Before percutaneous transmitral commissurotomy & $25.6 \pm 7.8$ & $24.9 \pm 8.5$ & 0.82 \\
independence & After 3 Months & $60.1 \pm 14.1$ & $61.3 \pm 12.1$ & 0.78 \\
Social Relationships & Before percutaneous transmitral commissurotomy & $22.8 \pm 6.2$ & $23.4 \pm 7.1$ & 0.72 \\
& After 3 Months & $58.3 \pm 10.3$ & $57.9 \pm 10.6$ & 0.66 \\
Environment & Before percutaneous transmitral commissurotomy & $30.9 \pm 6.9$ & $32.5 \pm 8.1$ & 0.64 \\
& After 3 Months & $58.6 \pm 7.9$ & $56.9 \pm 9.2$ & 0.83 \\
Spirituality & Before percutaneous transmitral commissurotomy & $35.1 \pm 9.2$ & $36.6 \pm 8.8$ & 0.74 \\
& After 3 Months & $58.9 \pm 8.6$ & $57.6 \pm 9.6$ & 0.91 \\
\hline
\end{tabular}


and $1.1 \pm 0.35$, after one month and three months of percutaneous transmitral commissurotomy respectively. The leaflet mobility of mitral valve is improved to $1.5 \mathrm{~cm}^{2}$ after which any further increase in MVA does not improve anterior and posterior leaflet mobility. ${ }^{17}$ This explains the subjective improvement and decrease in NYHA class after percutaneous transmitral commissurotomy in our subjects.

Our study showed that RVSP improved from $62.24 \pm$ 14.19 to $33 \pm 8.12$ after one month and $32 \pm 7.39$ after 3 months of follow up which was consistent with the results of different studies in which the RVSP decrease in the majority of the patients after percutaneous transmitral commissurotomy. ${ }^{18,19}$ Evidence from multiple international studies carried in different countries shows that the mitral valve area improves after percutaneous transmitral commissurotomy. ${ }^{16,20}$ Our results showed that mitral valve area improved from $0.8 \pm 0.67$ before percutaneous transmitral commissurotomy to $1.73 \pm 0.89$ after one month and $1.91 \pm 1.28$ after 3 months of follow up.

Khan et al. ${ }^{16}$ stated that quality of life improves after percutaneous transmitral commissurotomy but showed no subjective evidence to measure it. Ay et al. ${ }^{21}$ showed that quality of life improves after mitral valve repair more than mitral valve replacement showing that native valve has more favorable outcomes then prosthetic valves. Regardless of primary procedure whether surgical or percutaneous transmitral commissurotomy, when percutaneous transmitral commissurotomy was done secondarily for restenosis, there was no difference in relation to gender. ${ }^{22}$ Our results showed that quality of life improves after percutaneous transmitral commissurotomy when measured using WHOQol100 Questionnaire and it has significant correlation with net gain in mitral valve area with $R$ value of 0.46 and $p$ value of 0.03 . Furthermore, there is no significant group difference in relation to gender among all the six domains of WHOQol-100 questionnaire.

\section{Limitation}

Single centered study was carried out which cannot truly depict the trend in the general population, however, it will provide references for further multicenter studies and clinical trials. Quality of life was assessed subjectively through history and may have been subject to both patient and observer bias, however, the probability remains same for all the domains. Mitral Valve area was assessed by transthoracic echo which is operator dependent and has its own limitations however standard precautions were adopted to minimize the bias and results were confirmed from 3 experienced operators.

\section{Conclusion}

Successful percutaneous transmitral commissurotomy improves the quality of life in Mitral Stenosis patients. Quality of life has a positive correlation with a net gain in mitral valve area and there is no gender difference post-percutaneous transmitral commissurotomy in all the six domains of WHOQol-100 questionnaire.

\section{Conflict of interest}

None.

\section{References}

1. Nishimura RA, Otto CM, Bonow RO, Carabello BA, Erwin JP, Guyton RA. AHA/ACC guideline for the management of patients with valvular heart disease. The Journal of Thoracic and Cardiovascular Surgery:. 2014;148:e1-132.

2. Maken Z, Ahmed F, E- Elahi F, Arumghan A, Meher, Khatija. An epidemiological analysis of socioeconomic risk factors among patients of rheumatic heart disease, Islamabad. Pakistan. Pakistan Journal of Public Health. 2016;6:41-3, http://dx.doi.org/10.32413/pjph.v6i4.13.

3. Chopra P, Gulwari H. Pathology and pathogenesis of Rheumatic Heart Disease. Indian J Pathol Microbiol. 2007;50:687-95.

4. Sika-Pootanu D, Beaton A, Raghu A, Steer A, Carapetis J. Acute Rheumatic Fever and Rheumatic Heart Disease. [Acceso Mar 2017]. Disponible en: http://www.ncbi.nlm.nih.gov/ books/NBK425394.

5. Yildrim E, Celik M, Akpak YK. Mitral stenosis and pregnancy. Open Science J Clin Med. 2015;3:220-3.

6. Baumgartner H, Hung J, Bemejo J, Chambers JB, Evangelista A, Griffin BP. Echocardiographic assessment of valve stenosis: EAE/ASE. Recommendations for clinical practice. JASE. 2009;22:1-23.

7. Nawaz T, Jibran MS, Zahid ZU, Shawana, Gul AM. Immediate outcomes of percutaneous transvenous mitral commisurotomy in patients of rheumatic mitral stenosis. Pak Heart J. 2016;49:186-9.

8. Yeow WL, Kar S. Mitral stenosis; percutaeous transvenous mitral commissurotomy. Disponible en: http://www. thecardiologyadvisor.com/cardiology/mitral.stenosispercutaneous-transvenous-mitral-commissurotomy/article/ 583974/.

9. Tefera E, Leye M, Garceau P, Bouchard D, Miró J. Percutaneous transmitral balloon commissurotomy using a single balloon with arteriovenous loop stabilisation: an alternative when there is no Inoue balloon. Cardiovasc J Afr. 2018;29: 167-71.

10. Kannan M, Vijayanand G. Mitral Stenosis and Pregnancy; current concepts in anesthetic practice. Indian J Anaesth. 2010;54:439-44.

11. Rahman T, Rahman A, Majumaker AAL, Islam AKMM. TCTAPA-167 Percutaneous Transvenous Mitral Commissurotomy in patients with calcific mitral stenosis: immediate and in hospital clinical, echocardiographic and hemodynamic outcome. JACC. 2017;69 (16)supplement, http://dx.doi.org/10.1016/ j.jacc.2017.03.214.

12. Karimi M, Brazier J. Pharmaco Economics. 2016;34:645-9, http://dx.doi.org/10.1007/s40273-016-0389-9.

13. WHOQOL. Measuring Quality of Life. https://www.who.int/ healthinfo/survey/whoqol-qualityoflife/en/index4.html.

14. Li L, Young D, Xias S, Zhou X, Zhou L. Psychometric properties of the WHO Quality Of Life Questionnaire(WHOQol-100) in patients with chronic disease and their caregivers in China. Bulletin Of The World Health Organization. 2004;82: 492-502.

15. Movahed MR, Ahmadi-Kashani M, Kasravi B, Saito Y. Increased prevalence of mitral stenosis in women. J Am Soc Echocardiogr. 2006;19:911-3.

16. Khan I, Shah B, Habeel Dar M, Khan A, Faisal Iftekhar M, Sami A. Clinical and Echocardiographic Follow-up after Successful Percutaneous Transvenous Mitral Commissurotomy. Cureus. 2017;9:e1726, http://dx.doi.org/10.7759/cureus.1726. 
17. Hasan-Ali H, Shams-Eddin H, Abd-Elsayed AA, Maghraby MH. Echocardiographic assessment of mitral valve morphology after Percutaneous Transvenous Mitral Commissurotomy (percutaneous transmitral commissurotomy). Cardiovascular Ultrasound. 2007;5:48, http://dx.doi.org/10.1186/1476-7120-5-48.

18. Hamdy I. Assessment of right ventricular systolic function in patients with successful percutaneous mitral valve commissurotomy: The role of three-dimensional echocardiography. Heart Mirror J. 2011;5:356-9, http://dx.doi.org/10.1016/j.ihj.2014.02.012.

19. Zeb S, Ashraf T, Hashim M, Rizvi SNH. Regression of right ventricular systolic pressure after successful percutaneous mitral commissurotomy in patients with isolated severe mitral stenosis. Pakistan Journal of Medical Sciences. 2017;33:529-33, http://dx.doi.org/10.12669/pjms.333.12565.
20. Santosh Kumar C, Rajasekhar D, Vanajakshamma V, Boochi Babu M. The immediate and short term impact of successful percutaneous transvenous mitral commissurotomy on right ventricular function. J Cardiovasc Dis Diagn. 2015;3:217, http://dx.doi.org/10.4172/2329-9517.1000217.

21. Ay Y, Kara I, Aydin C, Ay NK, Inan B, Basel H, Zeybek R. Comparison of the Health Related Quality of Life of Patients Following Mitral Valve Surgical Procedures in the 6-Months Follow-up: A Prospective Study. Ann Thorac Cardiovasc Surg. 2013;19:113-9.

22. Sharma KH, Jain S, Shukla A, Bohora S., Roy B., Gandhi G.D., et al. Patient profile and results of percutaneous transvenous mitral commissurotomy in mitral restenosis following prior percutaneous transvenous mitral commissurotomy vs. surgical commissurotomy. Ind Heart J. 2014;66:164-8, http://dx.doi.org/10.1016/j.ihj.2013.12.007. 